\title{
Influence of tribology size effect on Stribeck curve in micro deep drawing
}

\author{
Hendrik Tetzel $^{\mathrm{a}}$ \\ Bremer Institut für angewandte Strahltechnik GmbH, 28359 Bremen, Germany
}

\begin{abstract}
The lubricant viscosity is one parameter for optimizing drawing processes. The influence is due to the velocity depending friction behaviour between work piece and tool, as well as the effective process forces and resulting pressures in the lubricant. Because of the tribological size effect, the knowledge gained in macro processes cannot be transferred directly to micro forming processes. To understand the influence of the drawing speed, several parameters as the drawing radius and viscosity of lubricants are varied in this work. The resulting Stribeck curves show a varying effect of the different kinematic viscosities. High viscosity lubricant reduces the occurring friction significantly at low Stribeck numbers, but increases friction with rising Stribeck number extremely. Concluding the results, it can be stated that the hydrodynamic friction demands a relevant portion of the overall friction in micro forming process especially at high drawing velocities, which cannot be explained by the lubricant pocket model.
\end{abstract}

\section{Introduction}

In recent years, a continuous trend towards miniaturization while improving the functionality of products can be observed in many industrial sectors. Especially sensors and actuators play an increasingly important role, so that the micro-system technology has gained importance [1]. As mechanical components of microsystems plastic parts are often used and their manufacturing in the micro range is already well studied [2]. An interesting alternative represent metallic micro components because they offer considerably different material properties and mass production by forming is relatively inexpensive. Due to these facts it seems very useful to scale down the size of existing forming processes as deep drawing, but this also leads to new challenges e.g. size effects [3]. Therefore, improvement and research of the micro deep drawing process is needed [4].

Because of the changed friction behaviour relatively high friction coefficients occur in micro forming processes compared to macro forming [5]. High friction causes higher energy consumption as well as higher tool wear and reduces the efficiency of the process. It results in higher punch forces in micro deep drawing processes [6].

Lubricants are made for optimizing the tribological behaviour of contact couples as separating them by establishing a lubrication film in between [7], corresponding to Stribeck-diagram. Function of lubrication films are reducing friction and wear also by transporting flakes out of the tooling, as well as transmission, cooling, damping, sealing and as corrosion protection.

\footnotetext{
${ }^{\text {a }}$ Corresponding author: tetzel@bias.de
}

This is an Open Access article distributed under the terms of the Creative Commons Attribution License 4.0, which permits unrestricted use, distribution, and reproduction in any medium, provided the original work is properly cited. 


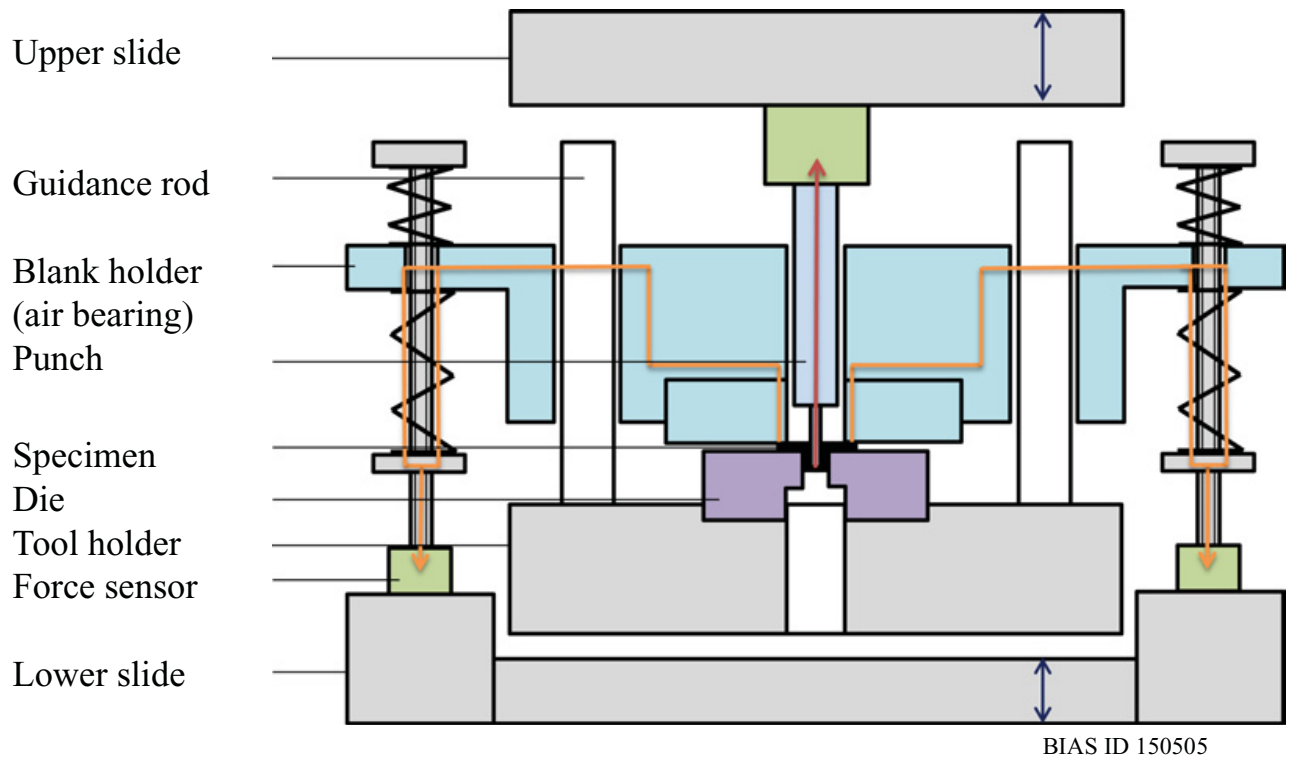

Figure 1. Experimental tool setup in micro forming machine with marked flow of forces (arrows).

A model explaining the size effects in lubricated friction was introduced first by [8]. But the lubricant pocket model gives just a qualitative description. In 2006, quantitative approaches were made by [9] with a mechanical-rheological model without considering viscosity of lubricant.

\section{Investigation methods}

\subsection{Micro forming machine}

In order to study the influence of lubricants viscosity on Stribeck curve, strip drawing experiments are conducted on a highly dynamic micro forming machine, which was engineered at BIAS [10]. Two individual moveable axis are present, driven by linear direct motors, which achieve maximum acceleration of $10 \mathrm{~g}$ each and reaching a maximum speed of $3.2 \mathrm{~m} / \mathrm{s}$, while the repositioning error is $1 \mu \mathrm{m}$ if the punch travel is less than $8 \mathrm{~mm}$, otherwise the positioning accuracy sticks to a $3 \mu \mathrm{m}$ error depending on the internal linear scales LC481 (Heidenhain GmbH).

\subsection{Tool and measurement system setup}

The die and blank holder are fixed in a tool holder, which is placed on the press bed in micro forming machine (cf. Fig. 1). A centred punch is connected directly to the force sensor, which monitors the punch force. The blank holder applies the blank holder force almost friction free, due to the use of pressurized air bearings, on the specimen. Springs with adjustable preload by threaded rods enhancing the control range and sensitivity of applied blank holder force on the specimen, measured by two single force sensors. Every force sensor is connected to a charge meter, which transmits the analogue force signal to an input device, which is filtering the signals to provide a feedback signal for machine control. For a proper process investigation, raw (unfiltered) measurement data are recorded at a sampling rate up to $1 \mathrm{MHz}$. The punch travel is recorded from linear scales by a quadrature encoder, simultaneously and saved together with the force values. 
Table 1. Testing parameters for experiments.

\begin{tabular}{ll}
\hline Constant process parameters & \\
\hline Punch width $\mathrm{w}_{\mathrm{p}}$ & $1.0 \mathrm{~mm}$ \\
Die width $\mathrm{w}_{\mathrm{D}}$ & $1.2 \mathrm{~mm}$ \\
Punch radius $\mathrm{r}_{\mathrm{P}}$ & $0.1 \mathrm{~mm}$ \\
Drawing gap $\mathrm{u}_{\mathrm{D}}$ & $0.1 \mathrm{~mm}$ \\
Initial sheet thickness & $0.05 \mathrm{~mm}$ \\
Initial blank holder pressure & $0.45 \mathrm{~N} / \mathrm{mm}^{2}$ to $0.8 \mathrm{~N} / \mathrm{mm}^{2}$ \\
Ratio (strip length/punch width) & 3 \\
Specimen material & $\mathrm{A} 199.5$ \\
Specimen dimension & $3 \times 4 \mathrm{~mm}^{2}$ \\
Tool material & $\mathrm{X} 153 \mathrm{CrMoV} 12$ \\
\hline Varied process parameters & \\
\hline Drawing radii $\mathrm{r}_{\mathrm{D}}$ in $\mathrm{mm}$ & $0.1 ; 0.3 ; 0.5$ \\
Drawing speeds $\mathrm{v}_{\mathrm{D}}$ in $\mathrm{mm} / \mathrm{s}$ & $10 ; 100$ to 1000 \\
Lubricants & low, medium and high viscosity lubricants \\
\hline
\end{tabular}

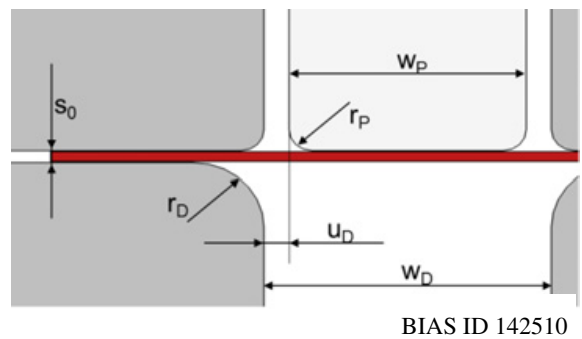

Figure 2. Geometric aspects for strip drawing test with double deflection-dimensions referred in Table 1 .

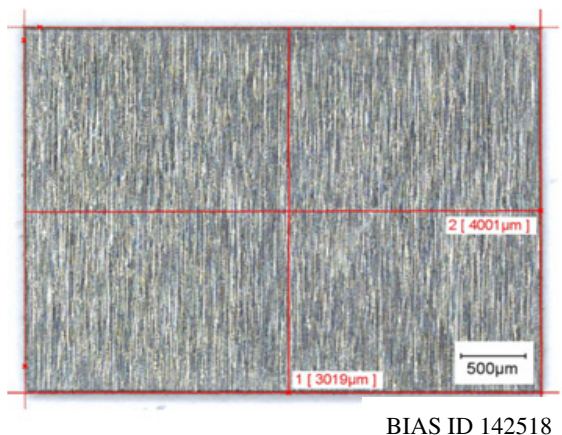

Figure 3. Specimen measured by Keyence digital microscope VHX-1000, rolling and drawing directions aligned.

Three dies with different drawing radii were applied, to vary the development of contact area between metal sheet and tool in drawing process. The setup of strip drawing test is shown in Fig. 2 . The geometrical parameters of the strip drawing experiments and the specimen are given in Table 1 With velocities between $10 \mathrm{~mm} / \mathrm{s}$ and $1000 \mathrm{~mm} / \mathrm{s}$ the punch is driven; in the range between $100 \mathrm{~mm} / \mathrm{s}$ and $1000 \mathrm{~mm} / \mathrm{s}$ an interval of $100 \mathrm{~mm} / \mathrm{s}$ is chosen. An initial blank holder pressure between $0.45 \mathrm{MPa}$ and $0.8 \mathrm{MPa}$ is loaded and controlled by micro forming machine. The adaptable initial blank holder pressure is to hold down the flange continuously. However, in the case of an excessive blank holder force, there is a risk of a one sided draw-in of the specimen and hence a failure, especially at low drawing velocities. 


\section{MATEC Web of Conferences}

Table 2. Properties of applied lubricants, AO - antioxidants, AW - anti wear, CI - corrosion inhibitors, EP - extreme pressure, FM - friction modifier, SE - surfactants and emulsifiers, VI - viscosity index improvers, WA - wetting agents.

\begin{tabular}{|c|c|c|c|c|}
\hline Lubricant & Base oil & $\begin{array}{l}\text { Density at } 15^{\circ} \mathrm{C} \\
\text { in } \mathrm{g} / \mathrm{cm}^{2}\end{array}$ & $\begin{array}{l}\text { Kinematic viscosity } \\
\mathrm{mm}^{2} / \mathrm{s}\end{array}$ & Additives \\
\hline High viscosity & Mineral & 0.96 & 400 at $40^{\circ} \mathrm{C}$ & AW, CI, EP, FM, VI, WA \\
\hline Medium viscosity & Vegetable & 0.93 & $\begin{array}{l}43 \text { at } 40^{\circ} \mathrm{C} \\
88 \text { at } 20^{\circ} \mathrm{C}\end{array}$ & $\mathrm{AO}, \mathrm{CI}, \mathrm{SE}, \mathrm{WA}$ \\
\hline Low viscosity & Ester & 0.84 & 52 at $20^{\circ} \mathrm{C}$ & AW, CI, EP, FM, SE, WA \\
\hline
\end{tabular}

\subsection{Specimens and data}

As work piece material A199.5 (EN AW1050A) with a thickness of $50 \mu \mathrm{m}$ is used. The blanks are cut by a Nd:YAG laser. The geometrical parameters of the cut strips are measured by a digital microscope VHX-1000 randomly, to ensure a width and length deviation of less than 1\% (cf Fig. 3). Before the specimens are used for forming process lubricant is applied by brush.

Every test series consists of seven single experiments combined with seven tests without specimens in order to isolate the true process forces from system inherent friction losses. Previously the raw data files are treated with a filter algorithm to cancel noise from measurement. The applied cut-off frequency and order of the low-pass filter algorithm were investigated before and depending on the process velocity.

Using the friction function (1) invented by [5], the punch force - punch travel diagrams are plotted to derive numerically a function of friction coefficient in dependency of contact pressure. The constants C1-C5 determine the character of the function.

$$
\mu=f(P)=C_{1}+C_{2} \cdot e^{-P \cdot C_{4}}+C_{3} \cdot e^{-P \cdot C_{5}} .
$$

Together with the initial blank holder pressure a friction coefficient for each experiment can be calculated. In order to present a Stribeck diagram the calculated friction coefficient is plotted over the specific Stribeck number, which consists of the kinematic viscosity $v$ multiplied by the drawing speed $\mathrm{v}$ and divided by the contact pressure $\mathrm{P}$.

\subsection{Lubricants}

To investigate the different influence of kinematic viscosities on Stribeck curve, three lubricants with dissimilar tribological behaviour are chosen (cf. Table 2). All three lubricants are typical for sheet metal forming application. After a testing session with one lubricant is completed, the tool setup is cleaned by using isopropyl alcohol thoroughly.

\section{Results}

A result from the drawing experiments Fig. 4 shows the comparison of punch force, punch travel curves at $v_{D}=1000 \mathrm{~mm} / \mathrm{s}$ punch velocity for drawing radius $r_{D}=0.1 \mathrm{~mm}$ of three applied lubricants. The curve of lubricant A indicates an almost $25 \%$ higher maximum punch force, than the recent ones in the range of punch travel between $0.25 \mathrm{~mm}$ and $0.5 \mathrm{~mm}$. Furthermore the general characteristic of the three curves is identical.

The difference between the process force diagrams using the three lubricants is less for other process parameters. Bigger drawing radii leading to smaller punch forces in general, while the high viscosity lubricant achieves relative small punch forces at low drawing velocities in process and vice versa.

In Fig. 5 the Stribeck diagram for a drawing radius of $r_{D}=0.1 \mathrm{~mm}$ is shown. Conspicuously high friction coefficients originate from low drawing speed experiments at $v_{D}=10 \mathrm{~mm} / \mathrm{s}$ and 


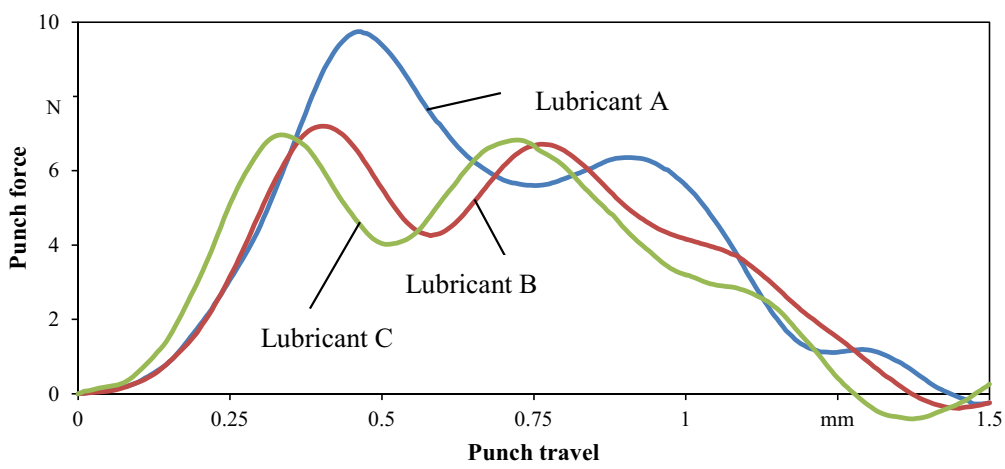

$\begin{array}{lr}\text { Specimen material: } & \text { A199.5 } \\ \text { Initial sheet thickness: } & 0.05 \mathrm{~mm} \\ \text { Drawing radius: } & 0.1 \mathrm{~mm} \\ \text { Drawing speed: } & 1000 \mathrm{~mm} / \mathrm{s}\end{array}$

Figure 4. Punch force-punch travel diagram for $r_{D}=0.1 \mathrm{~mm}$ and $v_{D}=1000 \mathrm{~mm} / \mathrm{s}$.
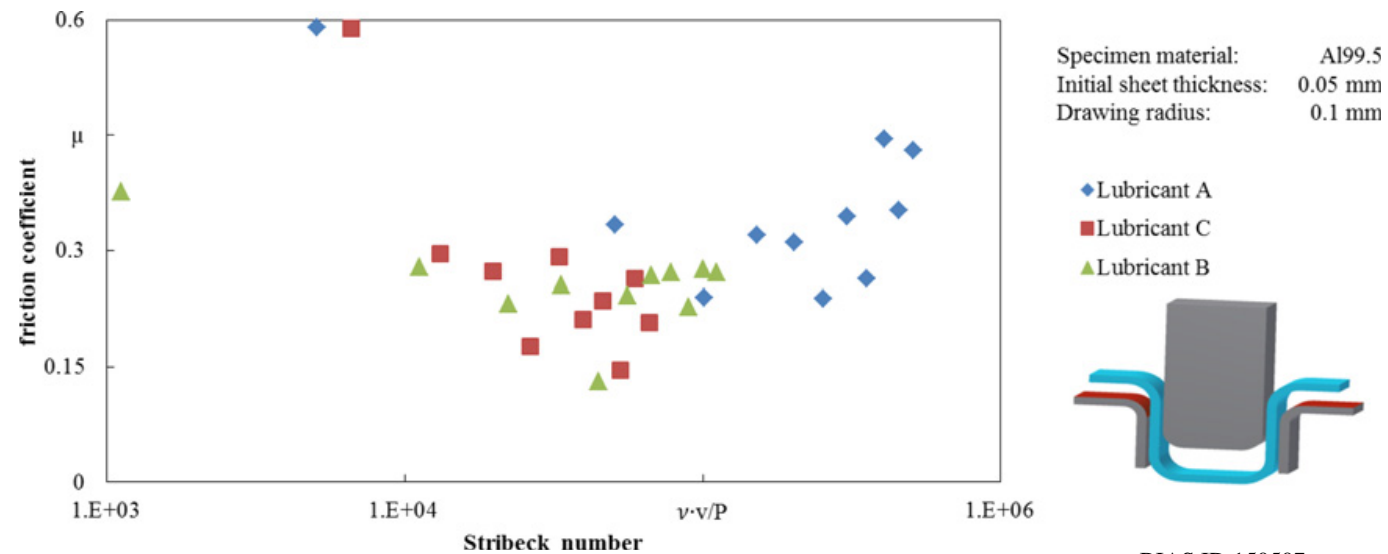

BIAS ID 150507

Figure 5. Stribeck diagrams for $r_{D}=0.1 \mathrm{~mm}$ with three different lubricants.

$\mathrm{v}_{\mathrm{D}}=100 \mathrm{~mm} / \mathrm{s}$. The characteristic of a typical Stribeck curve is shown in Fig. 5, low Stribeck numbers show highest friction. In the area between Stribeck numbers of $10^{4}$ and $10^{5}$ friction coefficients of 0.15 to 0.3 were primarily achieved by low and medium viscosity lubricants. On the right side of the diagram with Stribeck numbers higher than $10^{5}$ friction increases.

Stribeck diagrams for $r_{D}=0.3 \mathrm{~mm}$ and $r_{D}=0.5 \mathrm{~mm}$ are not depicted here, due to significant scattering of calculated friction coefficients, but the tendency is clearly towards higher friction for larger drawing radii.

\section{Discussions}

Concerning Fig. 4 the maximum punch force of high viscosity lubricant is significantly higher than for medium or low viscosity lubricant. This is due to the almost ten timer higher kinematic viscosity of the high viscosity lubricant. Especially for the small drawing radius, where the biggest contact area of the specimen is in contact to die and blank holder, the breakaway torque is clearly provable in the punch force, punch travel diagram. Furthermore the peaks of maximum punch forces are aligned in order of the kinematic viscosity. A possible explanation is that the low viscosity lubricant hinders the metal sheet less from entering the radius because of its rheological behaviour. 


\section{MATEC Web of Conferences}

Comparing the results of Stribeck diagram (Fig. 5) and the described curves of different drawing radii shows that larger drawing radii are leading to higher friction coefficients, but also gain scattering of friction coefficients - to data point reach friction values above the theoretical maximum of 0.577. Regarding the three areas of the Stribeck diagram (boundary, mixed and hydrodynamic friction) $\mathrm{r}_{\mathrm{D}}=$ $0.1 \mathrm{~mm}$ shows a relatively clear turning point of mixed to hydrodynamic friction at an approximately Stribeck number of $5^{10}$. A relevant factor for this issue of Stribeck diagram is the present pressure distribution in the contact area. As $\mathrm{Hu}$ [5] has published the non-uniform pressure distribution in the forming zone, there are only three areas of high pressure relevant for forming remain in process evaluation. If only the drawing radius is varied, a reduction yields to a higher change of shape of the material at a shorter stroke length. This increases the pressure load in the area of forming and hence the width of the area. Thus, this fact supports the lubricant pocket model. A larger width of high pressure contact encloses more lubricant and hinders it of pouring out. A better surface separation is obtained and lower friction is achieved. In opposite manner a larger drawing radius reduces the width of high pressure forming area, more lubricant pockets are open and the friction increase due to less surface separation. In comparison to macro scale the overall higher friction in micro deep drawing is a result of the geometrical size effect [11]. The roughness of contact surface is not scaled down by the specimen size. Consequently the count of appearing lubricant pockets is less in micro range.

Due to the fact, that the specimen width is constant for all used drawing radii, the initial support length of specimen on drawing die varies. For $r_{D}=0.1 \mathrm{~mm}$ it starts with $0.8 \mathrm{~mm}$ on each die side and ends with $0.4 \mathrm{~mm}$ on both sides for $\mathrm{r}_{\mathrm{D}}=0.5 \mathrm{~mm}$, therefore a different stationary lubrication status is achieved. The wide support area of the small radius $\left(r_{D}=0.1 \mathrm{~mm}\right)$ encloses a multiple count of closed lubricant pockets in contrast to the large radius, which is reducing friction significantly by separating the surfaces properly due to increasing internal hydrodynamic pressure. The less initial contact length between die and metal strip seems to be responsible for the scattering of calculated friction coefficients for larger drawing radii. A clear stationary lubrication is not achieved for small initial contact lengths.

The right side of Stribeck diagram (Fig. 5) shows increasing friction at higher Stribeck numbers. Lubricant with high kinematic viscosity induces hydrodynamic friction between die and metal strip with increasing relative velocity. This observation is in accordance with Stribeck, but not with lubricant pocket model. For low and medium drawing speed the lubricant pocket model provides an adequate interpretation of Stribeck diagram.

\section{Conclusions}

- The development of the stationary lubrication status depends on contact length and affects the friction coefficient significantly.

- A complete interpretation of Stribeck diagram in micro deep drawing is achievable by appliance of the lubricant pocket model and Stribeck's theory, due to the missing hydrodynamic formulation of lubricant pocket model.

The work reported in this paper is funded by the German research society (DFG) within the project "Punch velocity" (Vo530/42). The authors would like to thank the DFG for the financial support.

\section{References}

[1] J. Hesselbach, A. Raatz, J. Wrege, H. Herrmann, H. Weule, C. Buchholz, H. Tritschler, M. Knoll, J. Elsner, F. Klocke, M. Weck, J. von Bodenhausen, A. von Klitzing: mikroPro - Untersuchungen zum internationalen Stand der Mikroproduktionstechnik, wt Werkstattstechnik online, Bd. 93/3 (2003) 119-128 


\section{ICNFT 2015}

[2] U. Mescheder, Mikrosystemtechnik - Konzepte und Anwendungen, 2. Auflage, Teubner Verlag, Stuttgart (2004)

[3] G. Hirt, M. Bambach, H. Justinger, K. Zhao, Bedeutung von Größeneffekten für die MikroBlechumformung, Größeneinflüsse in Fertigungsprozessen, F. Vollertsen (Hrsg..), BIAS Verlag Bremen (2009) 117-134

[4] M. Geiger, M. Kleiner, R. Eckstein, N. Tiesler, U. Engel, Microforming, Annals of CIRP $\mathbf{5 0}$ (2001), 445-462

[5] Z. Hu, "Analyse des tribologischen Größeneffekts beim Blechumformen”, BIAS Verlag (2009)

[6] E. Doege et al., "Handbuch Umformtechnik", Springer Verlag Berlin Heidelberg (2010)

[7] H. Czichos et al., Tribologie Handbuch, Vieweg + Teubner (2010), 422

[8] O. Pawelski et al., Versuche und Berechnungen über das Ziehen und Einstoßen von Rundstäben, Stahl und Eisen 81(25) (1961), 1729-1739

[9] U. Engel, Tribology in Microforming, Wear, 260(3) (2006), 265-273

[10] H. Schulze Niehoff, Entwicklung einer hochdynamischen, zweifachwirkenden Mikroumformpresse, Strahltechnik Band 33, BIAS Verlag (2008)

[11] F. Vollertsen, Z. Hu, Tribological Size Effects in Sheet Metal Forming Measured by a Strip Drawing Test, CIRP Annals - Manufacturing Technology, 55, Issue 1 (2006), 291-294 